\title{
LYMPH NODE METASTASES IN PAPILLARY THYROID CANCER DETECTED BY QUANTITATIVE REAL-TIME POLYMERASE CHAIN REACTION FOR THYROGLOBULIN AND CYTOKERATIN 19
}

\author{
Krzysztof Kaczka ${ }^{1}$, Wojciech Fendler ${ }^{2}$, Maciej Borowiec ${ }^{2}$, Wojciech MŁynarski ${ }^{2}$, \\ AgnieszKa Celnik ${ }^{1}$, LeCH Pomorski ${ }^{1}$
}

\author{
1Department of General and Oncological Surgery, Medical University of Lodz, Lodz, Poland \\ Maria Sklodowska-Curie Memorial Hospital \\ 2Department of Pediatrics, Oncology, Hematology and Diabetology, Medical University of Lodz, Lodz, Poland
}

\begin{abstract}
Papillary thyroid cancer (PTC) metastases in the lymph nodes (LNs) were detected by real-time polymerase chain reaction (PCR) for TG and cytokeratin 19 (CK19), and the obtained results were compared with histopathology. 107 LNs from 34 PTC patients were divided into four blocks by a special cutting device -2 for histopathology, while the other 2 were tested by quantitative real-time PCR. Metastases were detected in 20 nodes from 10 (29.4\%) patients. TG and CK19 expression levels differed vastly between nodes with and without metastatic cells. ddCt of TG in the genetic material extracted from N0 nodes was $9.97 \pm 4.20$, while in nodes with metastases ddCt was $0.91 \pm 4.20(\mathrm{p}<0.0001)$. Cytokeratin 19 showed similar results with expression level (ddCt) in N0 nodes of $10.96 \pm 2.58$ vs. $7.73 \pm 3.63$ in nodes with metastases $(\mathrm{p}<0.0001)$. Evaluation of the utility of both parameters showed efficient differentiation of node involvement in the case of TG, with area under the ROC curve (AUC) equal to 0.91 (95\% CI: 0.85-0.96). Cytokeratin 19 also allowed for a degree of differentiation but its diagnostic efficacy was lower (AUC 0.76, 95\% CI: 0.640.88). The combined TG and CK19 quantitative real-time PCR could be used to select a previously missed group of patients with nodal involvement undetectable by standard histopathology.
\end{abstract}

Key words: thyroid cancer, lymph node metastases, quantitative real-time PCR for TG mRNA, quantitative real-time PCR for CK19 mRNA, histopathology.

\section{Introduction}

The impact of neck node metastases on the prognosis of patients with thyroid cancer is uncertain. Although ten-year survival is more than $90 \%$, according to some researchers, it is the worst prognostic factor, which decreases survival rate $[1,2]$. Nodal metastases in papillary thyroid cancer (PTC) are frequent (20-50\%), and up to $15 \%$ of the patients who undergo total thyroidectomy will develop a nodal relapse [3]. Other authors suggest that the presence of metastases in the regional lymph nodes has no clinical value [4-6]. The extent of lymphadenectomy is also a matter of controversy, especially in $\mathrm{cN} 0$ papillary thyroid cancer.

Selective lymphadenectomy or modified, radical lymph node excision could be considered in the case of differentiated thyroid cancer. There have been no randomized trials to date to offer guidance on this issue $[7$, 8 ]. Some surgeons suppose that the neck lymph nodes should be evaluated preoperatively and during surgery by physical and ultrasound examination. Only suspected lymph nodes have to be excised [7-9]. However, the 2006 guidelines of the American Thyroid Association recommended consideration of prophylactic bilateral cen- 
tral lymph node dissection for all thyroid cancer patients [10]. Total thyroidectomy with central lymphadenectomy does not increase the incidence of main complications: hypocalcemia and vocal cord paresis [11]. Sentinel lymph node biopsy has become more and more common in thyroid surgery but it is not a standard procedure like in breast cancer or melanoma [12-14].

It is necessary to perform many clinical studies to evaluate its prognostic value. We believe that to solve all these controversies there is a need to look for diagnostic methods other than histopathology. It seems that histopathology does not evaluate precisely the $\mathrm{N}$ status in some patients [15]. It has been recently shown that by slicing the lymph nodes into $2 \mathrm{~mm}$ tissue blocks and preparing one or more levels of hematoxylin-eosin (HE) staining from each block, as recommended by histopathological guidelines, metastases smaller than $2 \mathrm{~mm}$ might be missed [16]. Serious histopathology is more sensitive than conventional histopathology but it requires more intensive labor because a large number of histopathological specimens from each patient should be evaluated. That is why it is difficult to use it as a standard procedure. Immunohistochemistry is routinely used only in medullary thyroid cancer. In differentiated thyroid cancer, it is not so helpful. Some molecular examinations used for the assessment of lymph node status have shown higher positivity rates when compared to routine HE staining as well as immunohistochemistry. Nowadays lymph node metastases are diagnosed more and more often by molecular assays and immunohistochemistry [17-25]. The data of these studies may help to find more sensitive methods for the early detection of cell dissemination and may refine risk groups that might benefit from more extensive surgery or adjuvant therapy. These techniques are also performed in thyroid cancer. It has been proven that they could be useful for involved lymph node detection $[26,27]$. In this study we would like to look for the most suitable real-time PCR marker that could be used in differentiated thyroid cancer. Cytokeratin 19, RET, galectin-3 and HBME-1 expression in PTC were higher than in benign thyroid diseases, but they are not specific markers for PTC [28, 29]. The diagnostic efficiency of CK19 for PTC was slightly better than galectin-3 [30]. Earlier studies have proven that thyroglobulin is a suitable marker for real-time PCR in the evaluation of lymph node metastases in thyroid cancer $[26,31]$. In this study, the objective was to choose the best marker for molecular evaluation of lymph node metastases of PTC. To do this, real-time PCR examinations for TG and CK19 mRNA were performed and the obtained results were compared with histopathology as a gold standard.

\section{Material and methods}

Total thyroidectomy with appropriate lymphadenectomy was performed on the patients includ- ed in the study. The operations were performed according to the guidelines of the Polish Society of Surgeons and the Polish Society of Oncological Surgery [32].

The studied group covered 107 selected lymph nodes, randomized from 34 patients with papillary thyroid cancer. Histopathology examination of resected tumors revealed the classic subtype of papillary thyroid cancer to be most prevalent (32 patients), with two patients with oxyphilic subtype of PTC (6 nodes). The number of evaluated nodes from each individual ranged from 1 to 8 . The diameter of lymph nodes ranged from 7 to $13 \mathrm{~mm}$.

In all included lymph nodes TG and CK19 expression levels were measured and histopathological examination was performed.

To obtain representative material from all lymph nodes, they were divided into four blocks by a sterile, single use, special cutting device, primarily designed for one-step nucleic acid amplification (OSNA) examination [33].

\section{Quantitative real-time PCR procedure}

The reverse transcription reaction was performed using the commercially available set of High Capacity cDNA Archive Kit (Applied Biosystems, USA). cDNA was prepared from $1 \mu \mathrm{g}$ of mRNA isolated using the phenol-chloroform method (Tri Reagent, Sigma-Aldrich, St. Louis, MO, USA). For the reverse transcription reaction, random hexamer primers were used, according to the manufacturer's instructions $-10 \min 25^{\circ} \mathrm{C}, 2 \mathrm{~h} 37^{\circ} \mathrm{C}$, and $4^{\circ} \mathrm{C}$ thereafter. The resulting cDNA was diluted to a final concentration of $5 \mathrm{ng} / \mu \mathrm{l}$ and was used as a matrix in further experiments. The analysis of TG and KRT gene expression levels was performed using a human commercial available assay Hs00794359_m1 and Hs00761767_s1 (Applied Biosystems, Foster City, CA, USA), referenced with GAPDH expression level. The analysis was carried out in the genetic analyzer 7900HT Real-Time PCR (Applied Biosystems, Foster City, CA, USA). Comparative analyses of each of these genes in individual patients were performed using specialized computer programs SDS2.3 and RQ 2.1 (Applied Biosystems, Foster City, CA, USA). All amplification reactions were performed in duplicate. The mRNA expression levels of TG and KRT were calculated using the $2^{-\Delta \Delta C T}$ (comparative threshold $\mathrm{C}_{\mathrm{T}}$ 0.09) method, as detailed by the manufacturer.

\section{Results}

\section{Histopathology}

Metastases were detected in 20 nodes from 10 (29.4\%) patients. All of them were diagnosed with the classical subtype of PTC (Table I). 
Table I. Results of histopathology and quantitative real-time PCR

\begin{tabular}{|c|c|c|c|c|}
\hline LYMPH NODES & HISTOPATHOLOGY & REAL-TIME PCR TG MRNA & REAL-TIME PCR CK19 MRNA & $\mathrm{N}$ \\
\hline & negative & negative & negative & 79 \\
\hline & negative & negative & positive & 0 \\
\hline & negative & positive & negative & 8 \\
\hline & negative & positive & positive & 0 \\
\hline & positive & negative & negative & 4 \\
\hline & positive & negative & positive & 2 \\
\hline & positive & positive & negative & 9 \\
\hline & positive & positive & positive & 5 \\
\hline & total & & & 107 \\
\hline
\end{tabular}

Cut-off values with the best diagnostic efficacies were established at $d d C$ lower than 3.245 for TG and $d d C$ lower than 4.712 for CK19.

CK19-cytokeratin 19, TG-thyroglobulin

Table II. Diagnostic efficacy parameters of thyroglobulin and CK19 in detecting node involvement in patients with papillary thyroid cancer

\begin{tabular}{lcc}
\hline & TG $<3.245$ & CK19 $<4.712$ \\
\hline Accuracy & $88 \%$ & $86 \%$ \\
\hline Sensitivity & $78 \%$ & $35 \%$ \\
\hline Specificity & $91 \%$ & $100 \%$ \\
\hline Positive predictive value & $69 \%$ & $100 \%$ \\
\hline Negative predictive value & $94 \%$ & $85 \%$ \\
\hline CK19-cytokeratin 19, TG - thyroglobulin &
\end{tabular}

\section{Thyroglobulin and cytokeratin 19 expression results}

Thyroglobulin and CK19 expression levels differed vastly between nodes with and without metastatic cells. ddCt of TG in the genetic material extracted from N0 nodes was $9.97 \pm 4.20$, while in nodes with metastases ddCt was $0.91 \pm 4.20$ ( $<<0.0001)$. CK19 showed similar results with expression level (ddCt) in N0 nodes of $10.96 \pm 2.58$ vs. $7.73 \pm 3.63$ in nodes with metastases ( $p<0.0001)$. Levels of CK19 and TG were strongly correlated with each other $(\mathrm{R}=0.48 ; \mathrm{p}<0.0001)$, although the correlation was evident in samples without metastases $(\mathrm{R}=0.47 ; \mathrm{p}<0.0001)$ and absent in samples with metastases $(R=0.10 ; p=0.6623)$, suggesting the existence of distinct molecular subtypes.

Evaluation of the diagnostic utility of both parameters showed efficient differentiation of node involvement in the case of TG, with area under the ROC curve equal to 0.91 (95\% CI: 0.85-0.96). Cytokeratin 19 also allowed for a degree of differentiation but its diagnostic efficacy was lower (AUC 0.76 95\% CI: 0.64-0.88). Cutoff values with the best diagnostic efficacies were established at ddCt lower than 3.245 for TG and ddCt lower than 4.712 for CK19. Diagnostic efficacy parameters are shown in Table II.

The difference between the ROC curves was statistically significant in favor of TG $(p=0.006)$. Cy- tokeratin 19 exhibited potential for a highly specific diagnostic test, as all nodes $(\mathrm{N}=8)$ with $\mathrm{ddCt}<4.71$ contained metastatic cells. TG on the other hand had a higher detection rate of cancer (18 out of 23), but misclassified nodes as both false positive $(\mathrm{N}=8)$ and false negative results $(\mathrm{N}=5)$. The ROC curves for $\mathrm{TG}$ and CK19 are shown in Fig. 2.

Exclusion of nodes from patients with histopathological diagnosis other than the classic papillary tumor resulted in a marginal improvement of CK19 efficiency (AUC 0.81 95\% CI: 0.68-0.93) and no change in TG performance (AUC 0.90, 95\% CI: 0.84-0.96). Optimum diagnostic thresholds were however the same as in the whole sample.

\section{Discussion}

Lymph node status is the most important prognostic factor in resectable thyroid cancer. Patients with N0 status have much more favorable survival rates. However, many of them have relapse, despite surgical resection. This could be plausibly explained by an inadequate lymphadenectomy or inadequate histopathological examination. It seems that histopathology, eventually with immunohistochemistry, does not precisely evaluate the TNM status. In this study we tried to estimate lymph node status using quantitative real-time PCR. We hope that in the future molecular methods can better predict prognosis in patients with solid cancers. For example, in colorectal cancer, high risk of disease recurrence and poor survival in histopathological N0 lymph nodes was indicated based on molecular methods [34].

These patients could benefit from more radical treatment. In our study, concerning thyroid cancer, we wanted to compare two molecular examinations (real-time PCR for CK19 and TG) with each other as well as the standard histopathology. We chose TG because it seems to be one of the most specific substances for the thyroid. It is found only in normal thy- 


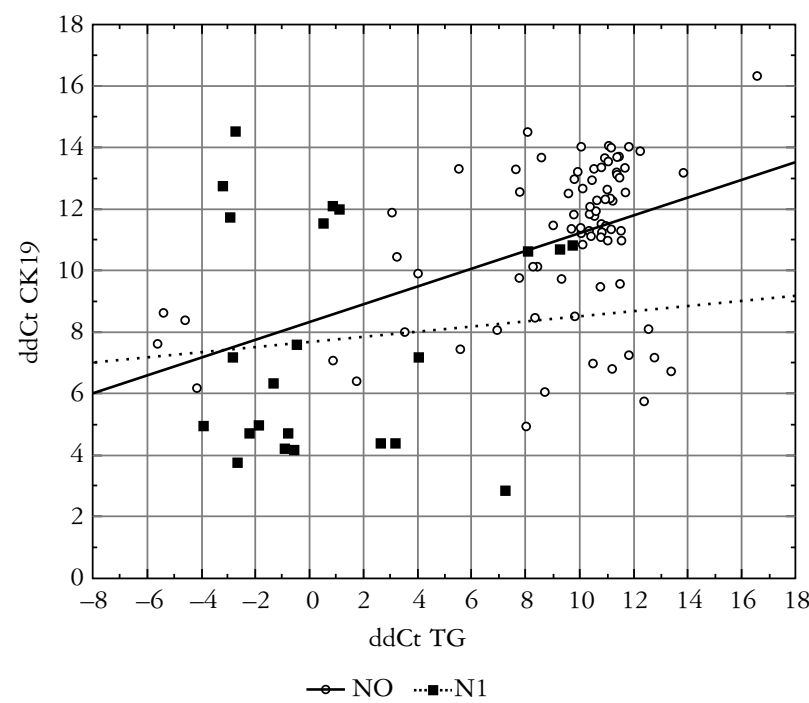

CK19-cytokeratin 19, TG-thyroglobulin

Fig. 1. Heterogeneity of CK19 and TG expression levels depending on nodal involvement

roid gland and cells of differentiated thyroid cancer, derived from follicular cells. Other markers, except thyroid peroxidase and NIS, are not so characteristic for thyroid gland [35].

Various low and high molecular weight cytokeratins, in general, are expressed differentially in papillary thyroid cancer [36, 37]. In particular, CK19 is demonstrated to be limited to papillary thyroid cancer, thus favoring the diagnosis of PTC in all its variant patterns [38].

Most of the previous studies have proposed CK19 as a useful marker that could be used to distinguish papillary thyroid cancer from other benign and malignant follicular lesions [39, 40]. In our study, we compared the results of histopathology with real-time PCR for CK19 and TG mRNA. According to our results if the preferred scenario would minimize node recurrence, TG could be used for screening using quantitative methods to select patients who should undergo lymphadenectomy due to an increased risk of node involvement. If reoperation can be performed safely, CK19 can be used for screening, selecting patients who are certain to show node involvement, with the rest requiring reevaluation after the histopathology workup. In the presented study TG showed a sensitivity of 88\%, suggesting potential for intraoperative screening if the molecular evaluation could be performed rapidly. An interesting observation was also made when the number of individual patients was analyzed. Out of the 5 missed cases of nodular involvement, 3 samples were collected from a single patient and had low expression of both CK19 and TG (a cluster of three nodes with positive results is clearly seen in the upper right quarter of figure 2 , suggesting a distinct molecular subtype

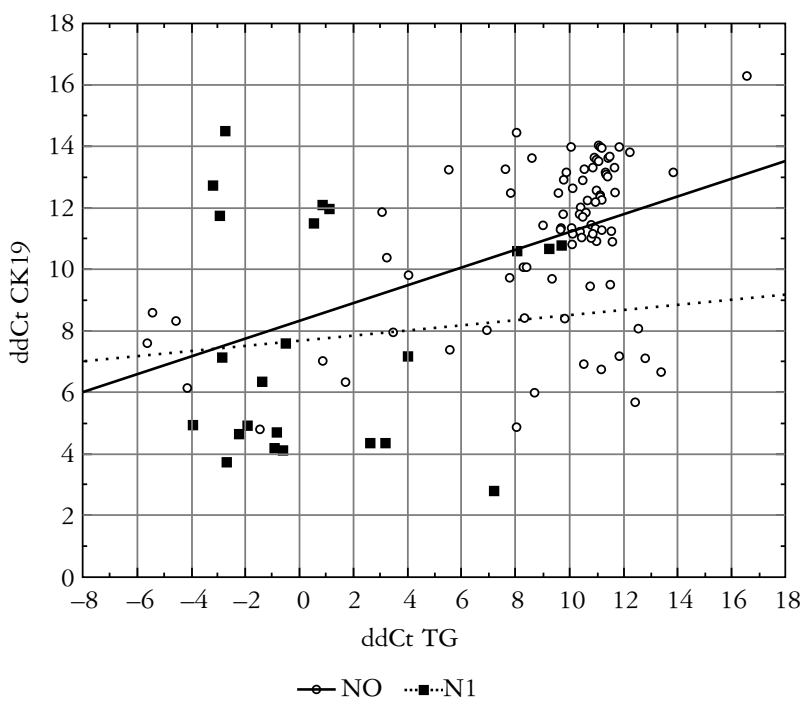

CK19-cytokeratin 19, TG-thyroglobulin

Fig. 2. ROC curves of TG and CK19 expression levels in lymph nodes suspected of harboring thyroid cancer metastases

in that patient. Moreover, this patient would be missed by both the TG and CK19 thresholds, indicating that molecular heterogeneity of thyroid cancers may pose unexpected challenges to expression-based screening for nodular metastases. False positive results suggested by molecular evaluation also deserve an explanation. Eight nodes with TG expression may in fact represent a group of nodes in which no evidence of metastases was found in histopathology due to the small number of present neoplastic cells picked up by expression level evaluation in real-time-PCR. If the latter scenario is true, molecular examinations could be used to select a previously missed group of patients with nodal involvement undetectable by standard histopathological examination. Follow-up examination of these putative false positive cases will show whether molecular or histopathological evaluation was clinically more important. Complex classification created using multivariate polynomial regression of combined expression scores of both CK19 and TG allowed us to increase the AUC to 0.95 and reduce the number of false positives to 4 . However, such classifiers would require additional computational steps and expression analysis of both genes doubles the workload. Our study has shown that quantitative real-time PCR for both markers TG and CK19, used in this study, is a fast, sensitive method of gene expression analysis. The application of validated probes reduces the risk of cross-reactivity. The method requires far less biological material than expression microarray analysis, uses fewer pre-analytic steps and is considerably cheaper. These qualities make it a feasible method for use in intraoperative examinations, provided that the hospital has rapid access to a genetic analyzer on standby. 
The study received financial support from the Ministry of Science and Higher Education in Poland - grant number N N403 220537.

Wojciech Fendler, Maciej Borowiec and Wojciech Mtynarski received financial support from the project "Polish Registry for Pediatric and Adolescent Diabetes - nationwide genetic screening for monogenic diabetes" financed by the Innovative Economy Operational Program.

\section{References}

1. Gagel RF, Goepfert H, Callender DL. Changing concepts in the pathogenesis and management of thyroid carcinoma. CA Cancer J Clin 1996; 46: 261-283.

2. Beasley NJ, Lee J, Eski S, et al. Impact of nodal metastaseson prognosis in patients with well-differentiated thyroid cancer. Arch Otolaryngolog Head Neck Surg. 2002; 128: 825-828.

3. Costa S, Giugliano G, Santoro L, et al. Role of prophylactic central neck dissection in cN0 papillary thyroid cancer. Acta Otorhinolaryngol Ital 2009; 29: 61-69.

4. Bhattacharyya N. A population-based analysis of survival factors in differentiated and medullary thyroid cancer. Otolaryngolog Head Neck Surg 2003; 128: 115-123.

5. Steinmüller T, Klupp J, Rayes N, et al. Prognostic factors in patients with differentiated thyroid cancer. Eur J Surg 2000; 166: 29-33.

6. Ward LS, Souza SL, Assumpção LV. The impact of nodal metastases on prognosis of well-differentiated thyroid cancer suggests the practice of prophylactic neck dissection. Arch Otolaryngolog Head Neck Surg 2003; 9: 495-496.

7. Cisco RM, Shen WT, Gosnell JE. Extent of surgery for papillary thyroid cancer: preoperative imaging and role of prophylactic and therapeutic neck dissection. Curr Treat Options Oncol 2012; 13: 1-10

8. Caglià $\mathrm{P}$, Zappulla E, Costa S, et al. Differentiated thyroid cancer: role of the lymph node dissection. G Chir 2010; 31: 293-295.

9. Kandil E, Friedlander P, Noureldine S, et al. Impact of extensive neck dissection on survival from papillary thyroid cancer. ORL J Otorhinolaryngol Relat Spec 2011; 73: 330-335.

10. Cisco RM, Shen WT, Gosnell JE. Extent of surgery for papillary thyroid cancer: preoperative imaging and role of prophylactic and therapeutic neck dissection. Curr Treat Options Oncol 2012; 13: 1-10.

11. Shindo M, Stern A. Total thyroidectomy with and without selective central compartment dissection: a comparison of complication rates. Arch Otolaryngol Head Neck Surg 2010; 136: 584-587.

12. Raijmakers PG, Paul MA, Lips P. Sentinel node detection in patients with thyroid cancer: a meta-analysis. World J Surg 2008; 32: 1961-1967.

13. Balasubramanian SP, Harrison BJ. Systematic review and meta-analysis of sentinel node biopsy in thyroid cancer. $\mathrm{Br} \mathrm{J}$ Surg 2011; 98: 334-344.

14. Kaczka K, Celnik A, Luks B, et al. Sentinel lymph node biopsy techniques in thyroid pathologies - a meta-analysis. Endokrynol Pol 2012; 63: 222-231.

15. Ece H, Devrim CD, Pelin TA, et al. Clinical experience of thyroid carcinoma: a study of 178 cases. Wspolczesna Onkol 2012; 16: $218-22$.

16. Weaver DL. The prognostic importance of isolated tumor cell clusters and micrometastases in sentinel lymph nodes. Cancer Invest 2009; 27: 121-128.

17. Ge MJ, Wu QC, Wang M, et al. Detection of disseminated lung cancer cells in regional lymph nodes by assay of CK19 reverse transcriptase polymerase chain reaction and its clinical significance. J Cancer Res Clin Oncol 2005; 131: 662-668.

18. Saintigny P, Coulon S, Kambouchner M, et al. Real-time RTPCR detection of CK19, CK7 and MUC1 mRNA for diagnosis of lymph node micrometastases in non small cell lung cancer. Int J Cancer 2005; 115: 777-782.

19. Taniyama K, Motoshita J, Sakane J, et al. Combination analysis of a whole lymph node by one-step nucleic acid amplification and histology for intraoperative detection of micrometastasis. Pathobiology 2006; 73: 183-191.

20. Chen G, McIver CM, Texler M, et al. Detection of occult metastasis in lymph nodes from colorectal cancer patients: a multiple-marker reverse transcriptase-polymerase chain reaction study. Dis Colon Rectum 2004; 47: 679-686.

21. Wascher RA, Bostick PJ, Huynh KT, et al. Detection of MAGE$\mathrm{A} 3$ in breast cancer patients' sentinel lymph nodes. Br J Cancer 2001; 85: 1340-1346

22. Merrie AE, Yun K, McCall JL. Detection of carcinoembryonic antigen messenger RNA in lymph nodes from patients with colorectal cancer. N Engl J Med 1998; 131: 662-668.

23. Denninghoff VC, Kahn AG, Falco J, et al. Sentinel lymph node: detection of micrometastases of melanoma in a molecular study. Mol Diagn 2004; 8: 253-258.

24. Demeure M, Doffek K, Komorowski R, et al. Adenocancer of the pancreas- detection of occult metastases in lymph nodes by polymerase chain reaction-bassed assay. Cancer 1998; 83: 1328-1334.

25. Samancilar O, Kaya SO, Ceylan KC, et al. The effect of TTF1 expression on ipsilateral mediastinal nodal (N2) metastases in primary adenocarcinoma of the lung. Wspolczesna Onkol 2012; 16: 516-519.

26. Kaczka K, Jakubiak-Wielganowicz M, Zawlik I, et al. Nodal status in the papillary thyroid cancer. Comparison of the results of routine histopathological examination, immmunohistochemistry and reverse transcription-polymerase chain reaction. Neoplasma 2008; 55: 513-518.

27. Weber T, Amann K, Weckauf H, et al. Detection of disseminated medullary thyroid cancer cells in cervical lymph nodes by cytokeratin 20 reverse transcription-polymerase chain reaction. World J Surg 2002; 26: 148-152.

28. Zhu X, Sun T, Lu H et al. Diagnostic significance of CK19, RET, galectin-3 and HBME-1 expression for papillary thyroid cancer. J Clin Pathol 2010; 63: 786-789.

29. Tong J, Wang Y, Da JP Usefulness of CK19, HBME-1 and galectin-3 expressions in differential diagnosis of thyroid papillary microcancer from benign lesions. Zhonghua Zhong Liu Za Zhi 2011; 33: 599-604.

30. Song Q, Wang D, Lou Y, et al. Diagnostic significance of CK19, TG, Ki67 and galectin-3 expression for papillary thyroid carcinoma in the northeastern region of China. Diagn Pathol 2011; 6: 126.

31. Pomorski L, Kaczka K, Piaskowski S, et al. Detection of lymph node metastases of papillary thyroid cancer-comparison of the results of histopathology, immunohistochemistry and reverse transcription-polymerase chain reaction-a preliminary report. Langenbecks Arch Surg 2005; 390: 209-215.

32. Herman K, Jarzab M. Endocrine tumors. In: Recommendations for diagnosis and treatment of malignant tumors. Krzakowski M (ed.). Via Medica, Gdańsk 2011; 358-373.

33. Tsujimoto M, Nakabayashi K, Yoshidome K, et al. One-step nucleic acid amplification for intraoperative detection of lymph node metastasis in breast cancer patients. Clin Cancer Res 2007; 13: 4807-4816.

34. Rahbari NN, Bork U, Motschall E, et al. Molecular detection of tumor cells in regional lymph nodes is associated with disease recurrence and poor survival in node-negative colorectal cancer: a systematic review and meta-analysis. J Clin Oncol 2012; 30: 60-70. 
35. Gubała E, Handiewicz-Junak D, Zeman M, et al. Thyroglobulin RT-PCR method for detection of lymph node metastases during the course of differentiated thyroid cancer. Wiad Lek 2001; 54: 349-356.

36. Raphael SJ, McKeown-Eyssen G, Asa SL, et al. High-molecular-weight cytokeratin and cytokeratin-19 in the diagnosis of thyroid tumors. Mod Pathol 1994; 7: 295-300.

37. Schelfhout LJ, Van Muijen GN, Fleuren GJ. Expression of keratin 19 distinguishes papillary thyroid cancer from follicular cancers and follicular thyroid adenoma. Am J Clin Pathol 1989; 92: 654-658.

38. Beasley MF, McLaren KM. Cytokeratin 19 and galectin-3 immunohistochemistry in the differential diagnosis of solitary thyroid nodules. Histopathology 2002; 41: 236-243.

39. Baloch ZW, Abraham S, Roberts S, LiVolsi VA. Differential expression of cytokeratins in follicular variant of papillary cancer: an immunohistochemical study and its diagnostic utility. Hum Pathol 1999; 30: 1166-1171.

40. Miettinen M, Kovatich AJ, Kärkkäinen P. Keratin subsets in papillary and follicular thyroid lesions. A paraffin section analysis with diagnostic implications. Virchows Arch 1997; 431: 407-413.

\section{Address for correspondence}

\section{Krzysztof Kaczka}

Department of General and Oncological Surgery

Medical University of Lodz

Maria Sklodowska-Curie Memorial Hospital

Parzęczewska 35

95-100 Zgierz, Poland

tel. +48503068786

e-mail:krzysztofkaczka@poczta.fm 